\title{
Article \\ Effect of Autoclave Pressure and Temperature on Consolidation of Layers and Mechanical Properties of Additively Manufactured (FDM) Products with PLA
}

\author{
Yousuf Pasha Shaik*(D), Jens Schuster, Aarif Shaik, Mustafa Mohammed (D) and Harshavardhan Reddy Katherapalli \\ Department of Applied Logistics and Polymer Sciences, University of Applied Sciences Kaiserslautern, \\ 66953 Pirmasens, Germany; y.shaik@gmx.de (J.S.); aash1001@stud.hs-kl.de (A.S.); \\ mumo0001@stud.hs-kl.de (M.M.); haka1002@stud.hs-kl.de (H.R.K.) \\ * Correspondence: yousuf.shaik@hs-kl.de
}

check for updates

Citation: Shaik, Y.P.; Schuster, J.; Shaik, A.; Mohammed, M.; Katherapalli, H.R. Effect of Autoclave Pressure and Temperature on Consolidation of Layers and Mechanical Properties of Additively Manufactured (FDM) Products with PLA. J. Manuf. Mater. Process. 2021, 5, 114. https://doi.org/10.3390/ jmmp5040114

Academic Editor: Steven Y. Liang

Received: 7 September 2021

Accepted: 20 October 2021

Published: 27 October 2021

Publisher's Note: MDPI stays neutral with regard to jurisdictional claims in published maps and institutional affiliations.

Copyright: (c) 2021 by the authors. Licensee MDPI, Basel, Switzerland. This article is an open access article distributed under the terms and conditions of the Creative Commons Attribution (CC BY) license (https:// creativecommons.org/licenses/by/ $4.0 /)$.
Abstract: In additive manufacturing technologies, fused deposition modelling (FDM) is continuing its advancement from rapid prototyping to rapid manufacturing. However, effective usage of FDM is not performed due to the poor mechanical properties of the 3D-printed components. This drawback restricts their usage in many applications. Much research, such as reinforcing 3D-printed parts with fibers, changing printing parameters (infill density, infill concentration, extrusion temperature, nozzle diameter, layer thickness, raster angle, etc.) are aimed to increase the mechanical properties of 3D-printed parts. This research paper aims to investigate the effect of pressure and temperature on the mechanical properties and consolidation of layers of 3D-printed PLA (Polylactic Acid). Posttreatment was done using a customized autoclave. Autoclave has the capability to maintain $185^{\circ} \mathrm{C}$ and 135 bar pressure. Three-dimensional-printed specimens were manufactured using the FDM process with two patterns. Later, the specimens were subjected to various post-treatment processes, then followed with testing and analysis of mechanical properties. Post-treatment process carried out by placing them in an autoclave at certain pressure and temperature conditions. To investigate the repeatability and tolerances, the test series includes a minimum of four to six test specimens. The results indicate that the concentric pattern yields the most desirable tensile, impact, and flexural strength due to the alignment of deposited rasters and better consolidation of layers with the loading direction. The pressure and temperature of the autoclave has a positive effect on the PLA samples, which helped them to reorganize the structure, hence strength properties were enhanced. The test results also compared with injection-molded samples for better understating.

Keywords: polylactic acid; 3D-print; injection mold; autoclave temperature pressurization; autoclave pressurization; oven temperature

\section{Introduction}

Additive manufacturing is also known as 3D printing; 3D printing is one of the rapidly growing plastic processes that involves fabrication of a CAD (Computer Aided Design)-model. It is available in various types such as fused deposition molding (FDM), stereolithography (SLA), powder bed fusion, material jetting, etc. In 1984, the first working 3D printer was developed by Charles W. Hull of 3D-Systems Corp, who named it Stereolithography apparatus, which was more expensive at that time. This technology has become a pastime of numerous designers, researchers, inventors, and similar who freely design and produce their own products. Due to the steady progress of 3D printing in the early 1980s, 3D printing was limited to several applications and designs, but its great value and potential was always evident for the manufacturing industry. In the past few years, 3D printing has made significant leaps forward, since technicians are continuously testing and identifying drawbacks that exist in the technology. 
The advantage of 3D printing now lies in its high productivity and flexibility, shorter production time, greater precision, a reduction in waste materials, overall cost reductions, improved product quality, and lightweight products [1]. The advancements in all types of additive manufacturing increase their chances for usage in many industries such as automobile, medical, construction, aerospace, and many other applications.

Among all other additive manufacturing techniques, fused deposition modelling (FDM) plays a dominant role in all kinds of industries from small-scale to large-scale due its low cost and its ease of operation, even by non-professionals [2]. FDM is a process in which a circular cross-sectional filament of predefined diameter is made to push into the hot end by means of a feeder with a defined speed, and melted material is exited from the nozzle attached to the hot end, which follows a defined path to construct a specimen layer-by-layer (Figure 1). The path and process parameters (printing patterns, infill density, infill angle, nozzle temperature, printing speed, etc.) can be given and controlled by a software called slicer using a 3D CAD model [3].

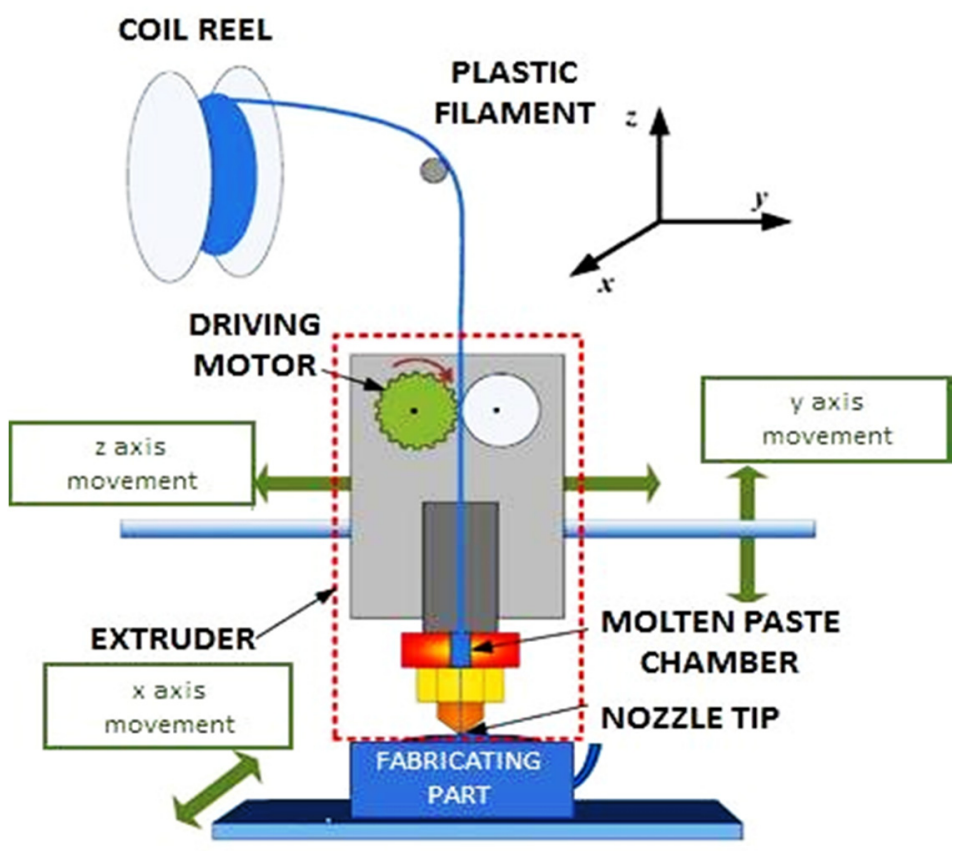

Figure 1. A diagrammatic representation showing the working of FDM/FFF 3D printing technology.

FDM is a cost-effective way to quickly produce prototypes and functional parts, aside from cheap machines and a simple technological process. Additionally, the supply of thermoplastic materials is widely available, facilitating prototyping and industrial usages. On the contrary, the technology has a few drawbacks that reduce its efficiency. When comparing the dimensional accuracy and resolution among the 3D-printed technologies, FDM possesses the lowest values. Moreover, an FDM model's visible layer lines and anisotropic characteristics require the post-processes part to achieve a smooth, flat surface [4].

Even though FDM rapid prototyping has wide range of applications, it is not used to produce structural parts due to poor mechanical properties of the 3D-printed parts. This drawback restricts their usage in many applications. At present, many research studies aim to increase the mechanical properties of 3D-printed parts. Some studies propose reinforcing the 3D-printed parts with fibers (short fibers or long continuous fibers) to improve their properties [5]. In one research study, SiC-coated PLA filaments used in the FDM process are an effective way to augment the mechanical properties. SiC-coated PLA composites were printed and heated in the microwave, which increases the remelting of layers, at $185{ }^{\circ} \mathrm{C}$ for $60 \mathrm{~s}$; in this way, the adhesion and consolidation of layers resulted in a high strength [6].

Research reveals that a typical FDM printer has been inserted into the nitrogen atmosphere to print parts that had shown a 30\% increment in the tensile strength [7]. Another 
research investigation specifies that heat treatment of 3D-printed parts enhances adhesion between the interlayers and reduces internal stresses [3]. However, an annealing process has constraints, as some polymers are temperature sensitive and may expose thermal shrinkage or warping. One of our earlier studies dealt with post-treatment processing for better compaction of the polymer material processed by FDM. Before placing them in an autoclave at high pressure, mechanical testing was done on 4 different patterns with different infill densities and chosen the best one for post-treatment. 3D-printed PLA samples were placed in a customized autoclave at a temperature under the glass transition range, with a pressure of 50 bars exposed for 10 hours. The effect of pressure and temperature on 3D-printed samples were analyzed. The combined effect of pressure and temperature, which relived internal stresses, augmented grain structure, enhanced their mechanical properties by approximately $20 \%$, and the results were published [3]. Another study revealed that 3D-printed FDM parts exhibit more enclosed voids, as compared to injection moulding-composed parts amid pressure during the process, and tight dimensional control [8]. These voids are in conjunction with mechanical strength. The pressure plays a significant role in controlling the isotropic behaviour of parts. The voids can be disciplined by reducing layer thickness and varying infill density. However, microvoids are available in such cases, which cannot be nullified completely.

Inadequate adhesion between the deposited layers and stacking layers while printing might be the cause of the poorest mechanical properties. This might be due to the temperature difference between the already deposited layers and incoming layers, as it relies on extruding the heated material and cooling [6].

In this research work, a customized autoclave and an oven were used to improve the mechanical properties of 3D-printed samples by post-processing treatment in different environment conditions (with pressure and temperature, only pressure, and only temperature conditions) and comparing its properties before and after post-processing.

\section{Materials and Methods}

\subsection{Material}

Polylactic acid (PLA) is a sugar-based biodegradable and environmentally friendly polymer. It is derived from any sugar plants available, such as corn starch, tapioca, or sugar canes. Its natural properties cause the material to degrade easily in three to six months. Moreover, plastic is widely used in fabric engineering, drug supply systems, and bone structure fixation due to the compatibility between PLA and the body [2]. It is simpler to print with PLA filament than others, since the material has a low melting temperature, between $180{ }^{\circ} \mathrm{C}$ and $220^{\circ} \mathrm{C}$. PLA offers a smooth appearance and is a perfect choice for aesthetic rather than functional products. As a result, PLA products are used in rapid prototyping or in other applications such as figures, low wear toys, and containers where high mechanical features are not required. Another advantage when printing with PLA is wrapping resistance, which is accounted for in the low thermal expansion coefficient [8]. Unlike ABS, which emit toxic fumes such as styrene, PLA is safe, and if ventilation is installed, it does not pose a danger to the human health.

PLA is a semi-crystalline or amorphous polymer with a glass transition temperature (Tg) and melting temperature (Tm) of approximately $55^{\circ} \mathrm{C}$ and $180{ }^{\circ} \mathrm{C}$, respectively. The thermal properties of PLA could be affected by different structural parameters, such as molecular weights and composition (stereoisomers content).

\subsection{Fabbmatic 3D Printer}

All the samples were printed with the Fabbmatic Mendelmax FM Pro Desktop 3D printer (Fabbmatic, Pirmasens Germany) [9], which uses fused deposition modelling printing technology (Figure 2). The printer filament was manufactured by RepRap World [10] with a diameter of $1.75 \mathrm{~mm}$. This generation printer was connected to a smart extruder whose nozzle diameter is $0.4 \mathrm{~mm}$. The 3D design of the test piece was first converted to and saved as an .STL file, a file format that stores information about one or multiple 
3D models' location, orientation, and other printing settings in the Fabbmatic Desktop Software (pronterface, San Francisco, CA, USA). It was then exported to the Slic3r program to open; the STL file was then exported in .gcode file type to be interpreted by the printer.

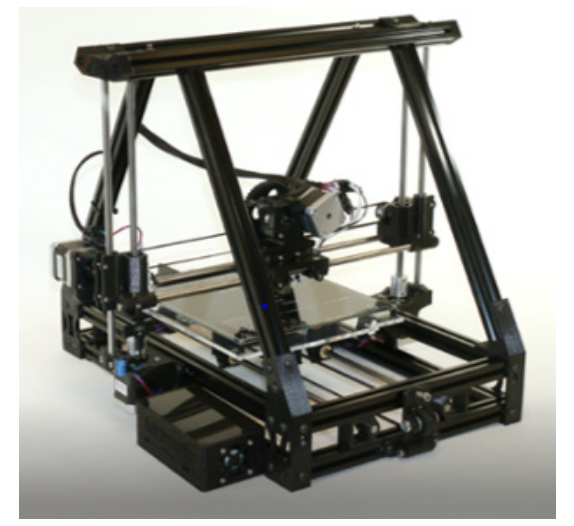

Figure 2. Fabbmatic Mendelmax 1.5 FM Pro Desktop 3D Printer [11].

All the samples were designed by the Solid works (2019) student edition with the actual shape and dimensions according to the standards required for mechanical testing and has been exported to a STL format, so that it can be read and interpreted by the printing parameterization software. In this project, the Slic3r (open-source 3D printing toolbox) software has been used, from which the .gcode file is obtained for printing. All the specimens were printed using Fabbmatic (Mendelmax FM Pro).

\subsection{Customized Autoclave}

An autoclave is a machine Figure 3 that is used to perform industrial and scientific procedures that require higher temperatures and pressures than the ambient pressure and temperature. By sterilizing bacteria, viruses, and fungus, autoclaves are employed in medical applications to inactivate them [12].

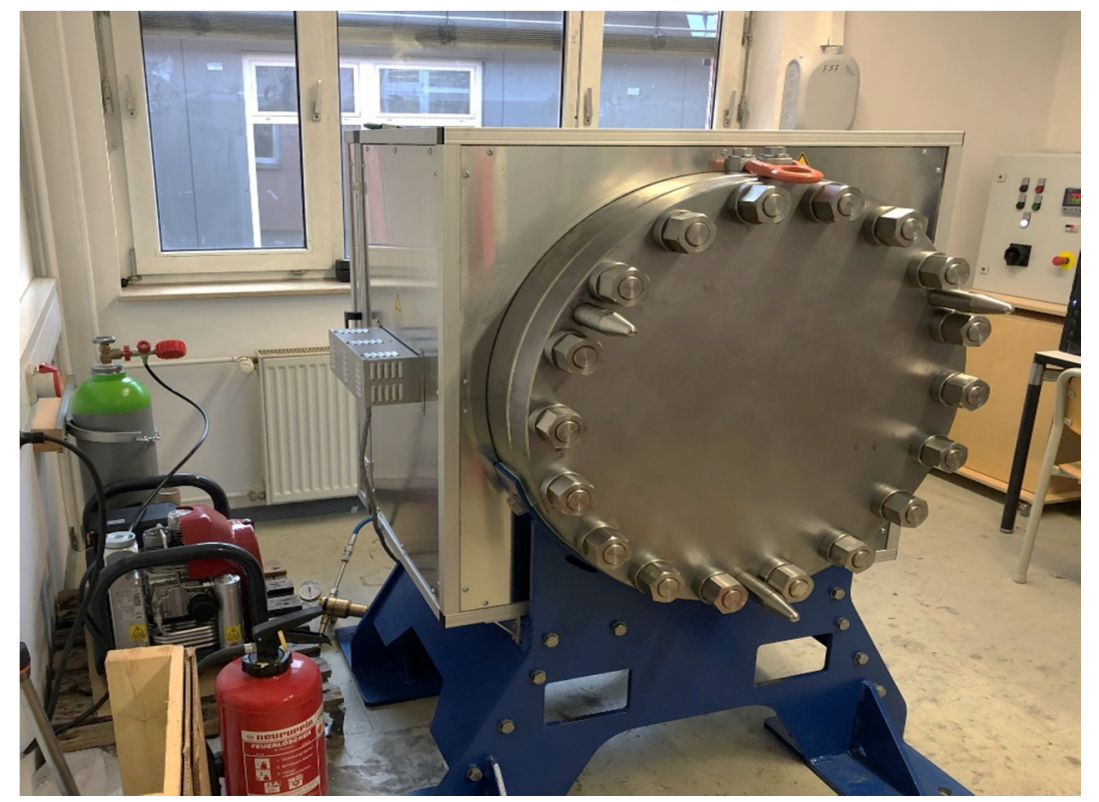

Figure 3. Autoclave setup in the laboratory.

They are employed in the chemical industry to treat coatings, vulcanize rubber [13-24], and perform hydrothermal synthesis. Autoclaves used in laboratories or for research. With the option to select varied temperatures and times ideally suited for loads, autoclaves 
are specially intended to sterilize a wide range of sterilizing loads [14]. In industrial manufacturing, the structures are moved into warehouse-sized ovens and autoclaves after being piled and molded into the shape of a fuselage. The layers fuse together to produce a durable, aerodynamic shell [15-23].

Therefore, it was decided to test the PLA of 3D-printed and injection-molded samples in autoclave. It is important to maintain the ambient temperature and pressure, which is easily possible in an autoclave. The post-treatment process aimed to improve the mechanical properties of PLA by placing the 3D-printed specimens and injection-molded specimens inside the autoclave chamber under the desired pressure and temperature for a prescribed duration of time.

The autoclave setup which we used for our project work has been illustrated in Figure 3 below.

\subsection{Drying Oven for Temperature Condition}

In this research study, post-treatment of 3D-printed samples was performed in an oven, which is a Heraeus drying oven. Normally, oven annealing is easy to use where heat propagates from polymer component surface to the interior. However, for most engineering thermoplastic polymers such as PLA, due to the low thermal conductivity and thermal diffusivity, oven heat may take a long time to diffuse inside, which makes process energy-consuming from a practical perspective [16].

\section{Samples Preparations}

The research work aims to investigate three main mechanical strength properties, namely ultimate tensile strength, flexural strength, and impact strength in two different patterns (rectilinear and concentric).

Here, two types of sample structures, with respect to standards of the International Institute of Standardization, are used in this work.

A. ISO 527 Type 1A tensile specimen, for tensile test with a length of $150 \mathrm{~mm}$ and $4 \mathrm{~mm}$ thickness, is used as shown in Figure 4. All the tensile testing specimens in both 3D printing and injection molding are manufactured with in this standard.
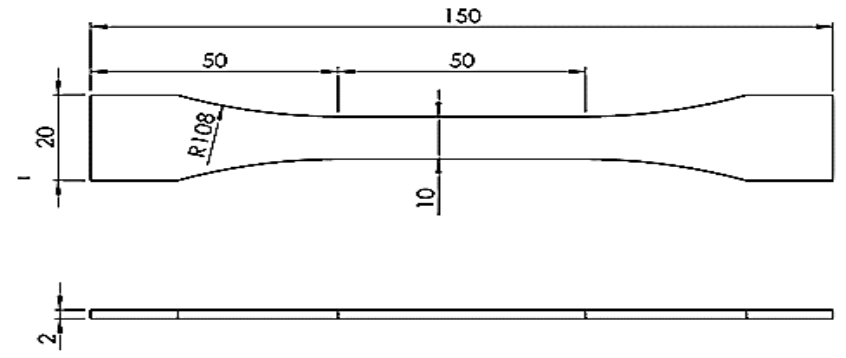

Figure 4. ISO 527 Type 1A sample dimensions.

B. DIN EN ISO 75 Rectangular form, for flexural and impact test, is required to be within the standard dimensions shown in Figure 5 below.
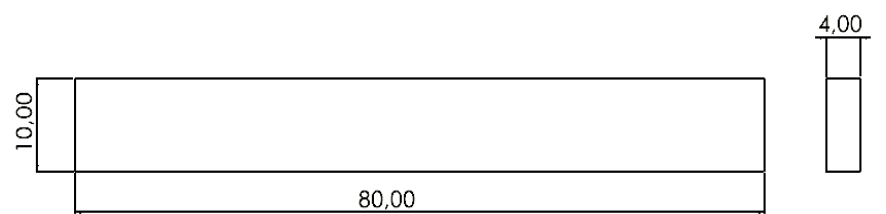

Figure 5. DIN EN ISO 75 sample dimensions.

\subsection{Printing Parameters for 3D-Printed Specimens}

Operators can achieve a desired-qualities part by adjusting numerous printer parameters such as built orientation, layer height, infill, printing speed, or the nozzle and build 
Platform temperature. Different settings can have different impacts on the results, so the operators should consider which options are more suitable for their products. For instance, the smaller the layer height is, the smoother and the more accurate the printer object becomes. This suits the parts whose shapes are complex and curved or those parts that require a smooth finish. However, an increased layer height reduces the total production time, and it is also cost-effective. The general FDM layer height ranges between $0.05 \mathrm{~mm}$ and $0.4 \mathrm{~mm}$, in which the value of $0.2 \mathrm{~mm}$ is commonly applied. Furthermore, eliminating support leaves a mark or rough surface on post-treatments, which may necessitate more work [17].

The slicer, a computer slicing software that converts a 3D object model, which is in the dimensions of ISO 527 (dog bone) and DIN EN ISO 75 (rectangular form), into specific instructions for the printer in most 3D printing procedures. In fused filament fabrication and other comparable processes, there is a conversion from a model in .STL format to printer commands in .g-code format.

The inner composition of the printed item, which can range from $0 \%$ to $100 \%$, is referred to as infill. It plays an important role in determining the modulus and strength of the whole feature. Higher infill means the part is denser or more solid, allowing the part to process a better mechanical strength. So, 100\% infill was chosen to print all the testing specimens. Infill patterns are also available in various shapes, including concentric and rectangular ones. A total of 100 samples for tensile, Charpy, and flexural testing were produced via 3D printing (according to Table 1). A nozzle of $0.4 \mathrm{~mm}$ was used for the 3D printer extruder. Around 60 samples were printed as shown in Figure 6, including dog bone and rectangular panels. After 3D printing, the test samples were exposed to autoclave temperature, autoclave pressure, and oven temperate.

Table 1. Printing parameters used in the experiments.

\begin{tabular}{cccccc}
\hline $\begin{array}{c}\text { Sample } \\
\text { Shape }\end{array}$ & $\begin{array}{c}\text { Infill } \\
\text { Patterns }\end{array}$ & $\begin{array}{c}\text { Nozzle } \\
\text { Temperature }\end{array}$ & $\begin{array}{c}\text { Infill } \\
\text { Percentage }\end{array}$ & $\begin{array}{c}\text { Weight in } \\
\text { Grams }\end{array}$ & $\begin{array}{c}\text { Printing } \\
\text { Time in } \\
\text { Minutes }\end{array}$ \\
\hline ISO 527 & Rectilinear & 230 & $100 \%$ infill & 10.31 & $26: 11$ \\
\cline { 2 - 6 } Dog bone & Concentric & 230 & $100 \%$ infill & 9.34 & $19: 20$ \\
\hline DIN EN & Rectilinear & 230 & $100 \%$ infill & 4.06 & $16: 29$ \\
\cline { 2 - 6 } ISO 75 & Concentric & 230 & $100 \%$ infill & 3.71 & $15: 34$ \\
\hline
\end{tabular}
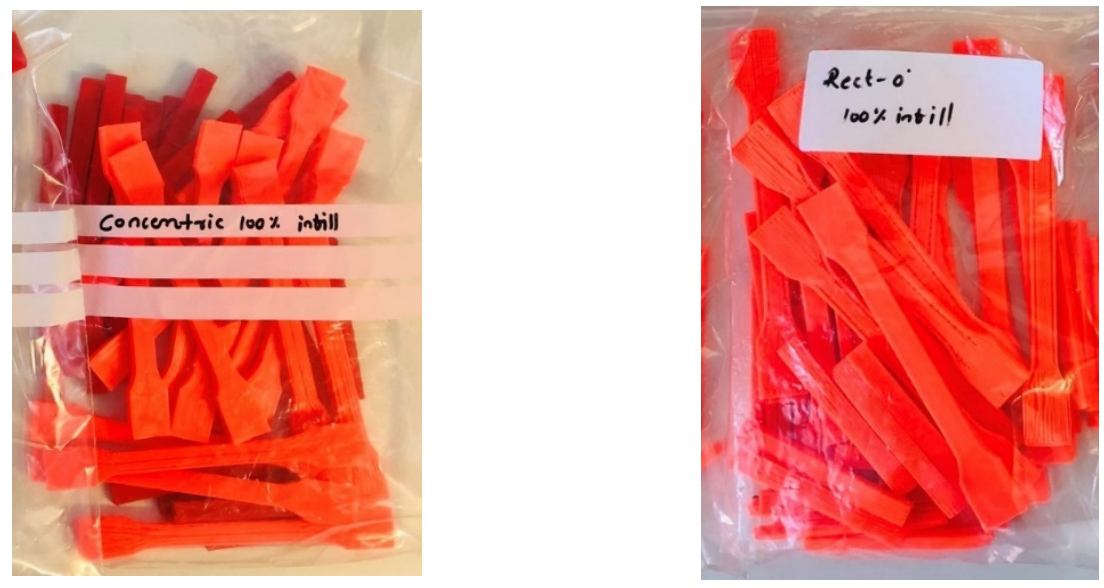

Figure 6. 3D-printed samples.

Sample preparation 3D printing process are presented in Figure 7. The testing sample was designed on the SolidWorks 2020 software (Dassault Systèmes SolidWorks Corporation, 
Waltham, MA, USA) according to the standard of ISO 527 (dog bone) and DIN EN ISO 75 (rectangular form).


Figure 7. 3D printing of DIN EN ISO 75 samples. (A-while printing, B-Printed Samples).

\subsection{Injection Molded Samples Preparation}

Injection molding process illustrated in Figure 8 is one of the most common process for manufacturing plastic parts in large volumes. In the injection molding process, the chopped filament PLA pellets [18] were used as the raw material. In this process, when the raw material was taken, the automatic Hooper and the required melting point temperature for the plastic material were given as the input to the machine. The proper mold temperature, mold cavity pressure, holding pressure, flow speed, and volume were properly uploaded, and then the PLA dog bone samples were manufactured [19].

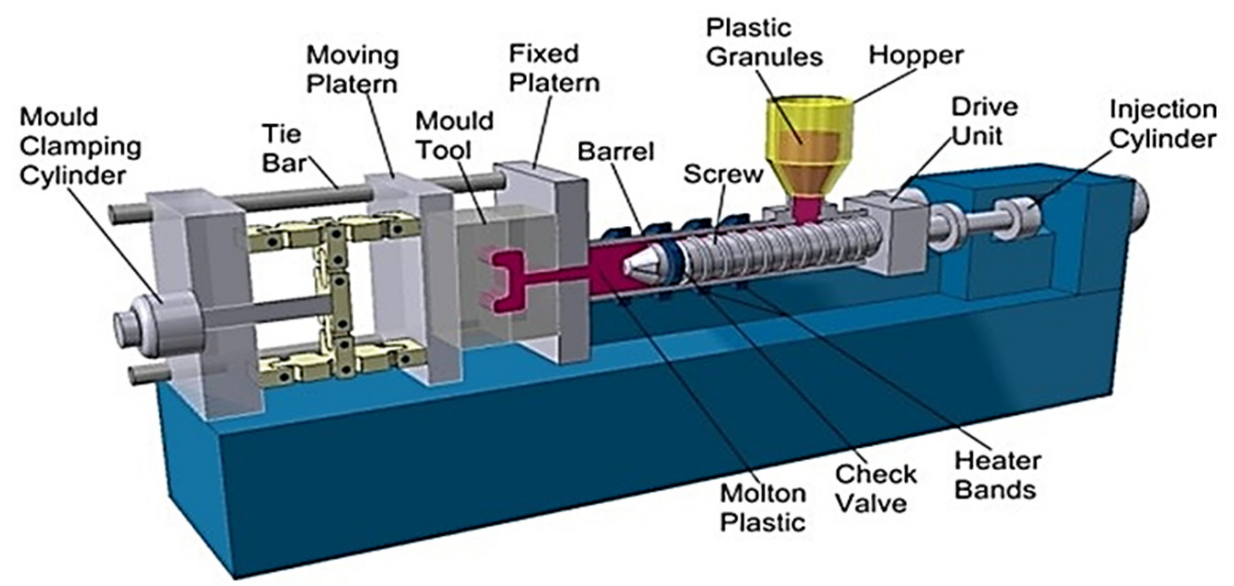

Figure 8. Schematic representation of the single screw injection molding machine.

In this research work, injection-molded specimens were manufactured using a $15 \mathrm{t}$ injection molding machine (Arburg, Lossburg, Germany) with cavity pressure and melt temperature measurements [20]. PLA is the material used. PLA filament is granulated, dried, and manufactured as a raw material for injection molding. Around 80 samples of PLA material were manufactured to draw the comparison with 3D-printed samples under different environment conditions. 


\subsection{Post-Treatment Process of Testing Specimens}

3.3.1. Parameters of Autoclave Pressurization with Temperature Process

The 3D-printed samples of concentric patterns and injection-molded specimens were treated inside the autoclave between temperatures $30^{\circ} \mathrm{C}$ and $55^{\circ} \mathrm{C}$, pressure was gradually increased from 0.1 bar to $15 \mathrm{bar}$, and two successive trails were performed each at 5 bar and 15 bar. In Figure 9 and Table 2 the trails and parameters of autoclave pressurization with temperature of both injection-molded and 3D-printed PLA samples are presented. Pressure is maintained by a pre-charged cylinder with compressed air (up to 20 bar for $15 \mathrm{~min}$ ). Pressure and temperature are controlled using a Haage monitor [21].

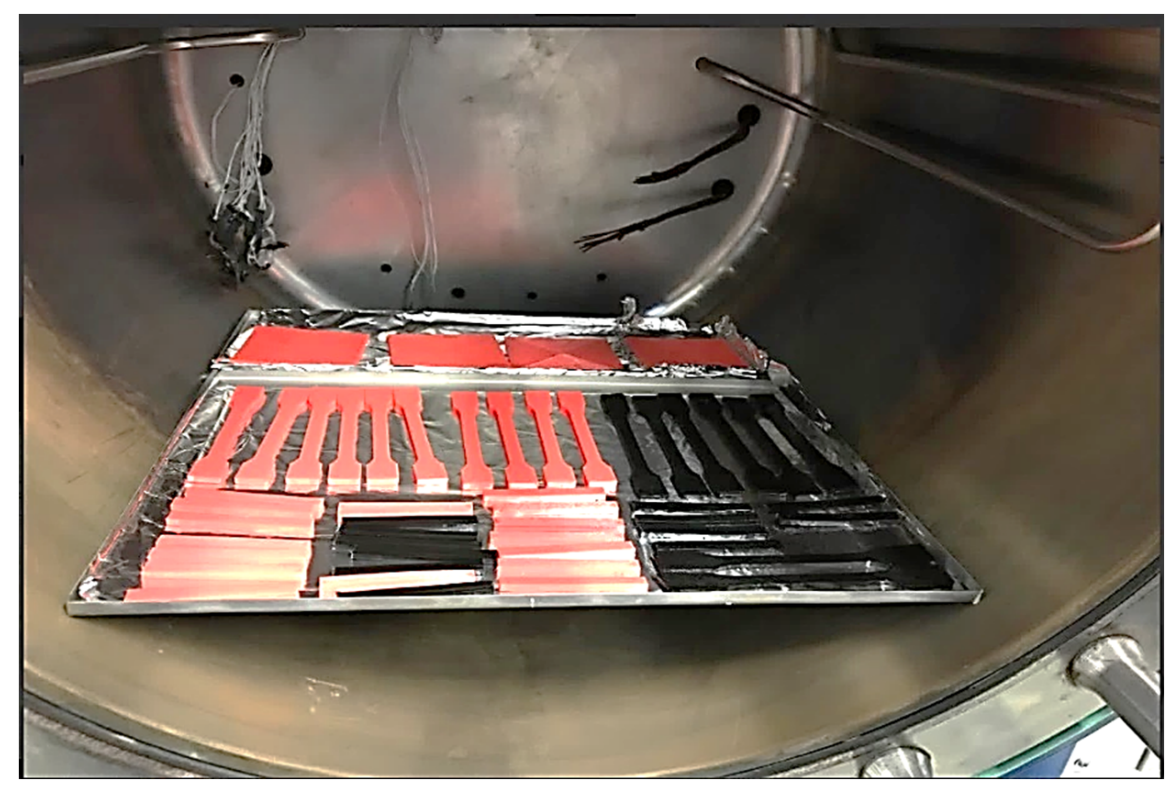

Figure 9. 3D-printed and injection-molded samples autoclave arrangement.

Table 2. Showing trails and parameters of autoclave pressurization with temperature of PLA samples, both injection-molded and 3D-printed.

\begin{tabular}{cccc}
\hline Number & Pressure & Temperature & Duration \\
\hline Trail-I & $5 \pm 0.5$ bar & $30-34{ }^{\circ} \mathrm{C}$ & $2 \mathrm{~h}$ \\
Trail-II & $15 \pm 0.5$ bar & $45-55.2{ }^{\circ} \mathrm{C}$ & $8 \mathrm{~h}$ \\
\hline
\end{tabular}

\subsubsection{Parameters of Autoclave Pressurization (Temperature Maintained at $25^{\circ} \mathrm{C}$ )}

The 3D-printed samples of concentric and rectilinear pattern, both with $100 \%$ infill printed specimens, were treated inside the autoclave as shown in Figure 9 pressure from 0.1 bar to 50 bar by gradually increasing the pressure, and temperature was maintained at room temperature, that is, $25^{\circ} \mathrm{C}$. The maximum pressure was attained after $2 \mathrm{~h}$. After the 50-bar pressure was attained in the autoclave, it was left for $8 \mathrm{~h}$ for post-processing of samples under this pressure condition.

\subsubsection{Oven Temperature Treatment on FDM Samples}

The 3D-printed samples of concentric and rectilinear pattern, both with $100 \%$ infill print specimens, were annealed at $60{ }^{\circ} \mathrm{C}$ temperature inside the oven, as shown in Figures 10 and 11, with 0 bar pressure for $8 \mathrm{~h}$. 


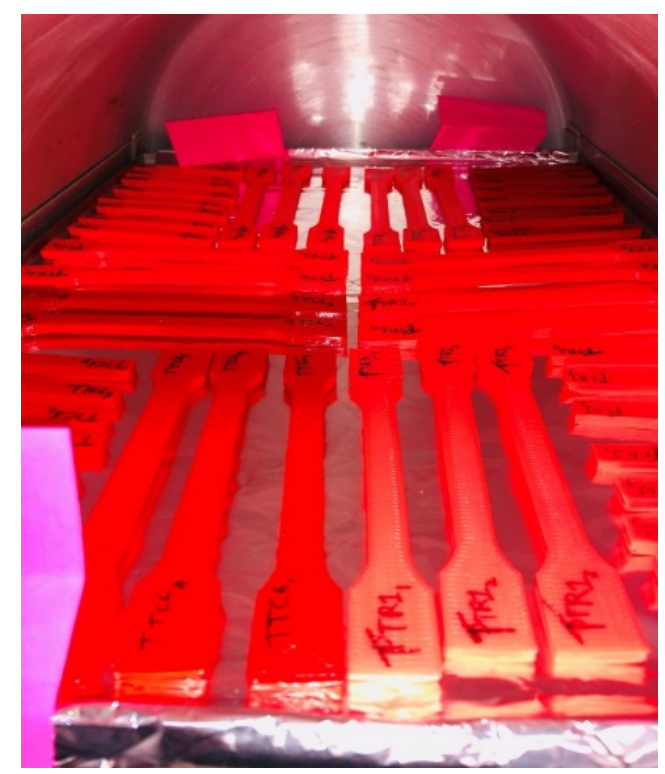

Figure 10. 3D-printed samples annealed in the oven.

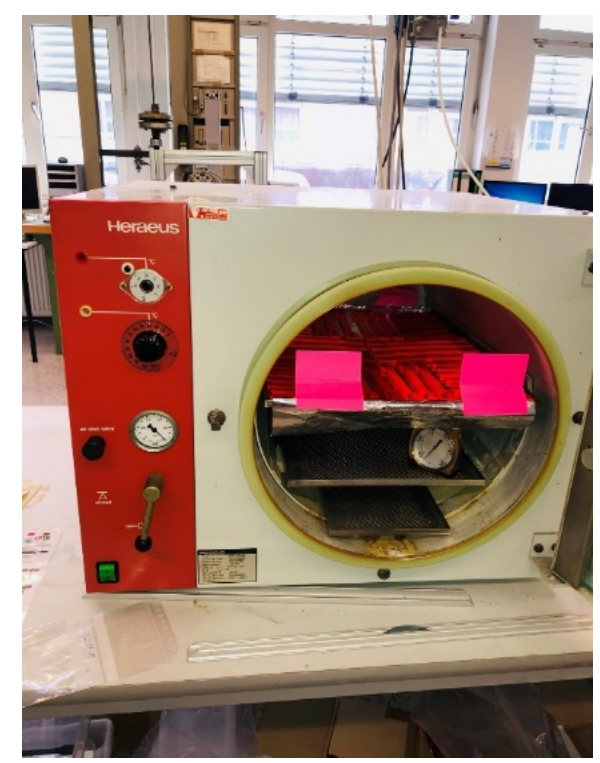

Figure 11. Oven at $60^{\circ} \mathrm{C}$ and air pressure at 0 bar.

\section{Results and Discussions}

4.1. Comparing the Test Results of Injection Molded PLA before and after Autoclave Temperature and Pressure Treatment

A comparison is made between PLA injection-molded samples and autoclave temperature- and pressure-treated PLA.

From the graph in Figure 12, it is clear that there is significant increase (almost 30\%) in tensile modulus of injection-molded samples after the post-treatment in autoclave under temperature and pressure conditions. Additionally, there is an increase in flexural modulus and impact strength after post-treatment in the autoclave but comparatively less improvement than the tensile modulus. 


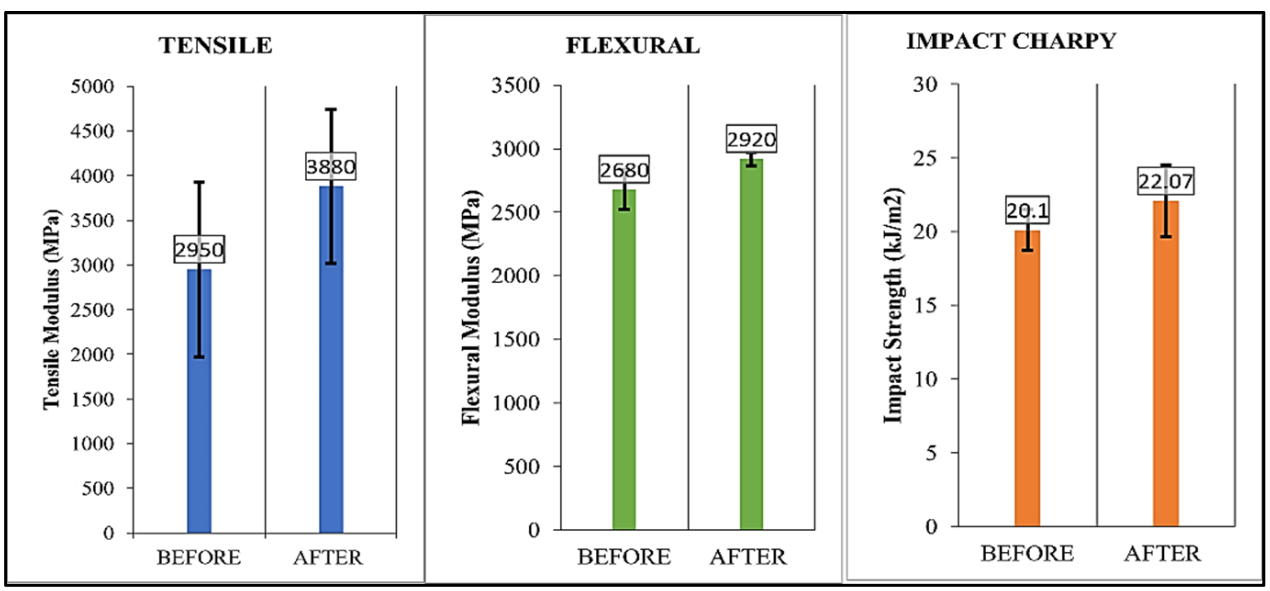

Figure 12. The change in modulus and strength after autoclave temperature pressurization.

\subsection{Comparing Strengths of 3D-Printed PLA before and after Oven Temperature Treatment} 4.2.1. Tensile Test Results

The result of a tensile test displays the tensile modulus and weight difference of the 3D-printed samples shown in Figure 13; there was, on general, a $0.2 \%$ reduction in weight of the specimens after the oven temperature process. Both patterns, concentric and rectilinear, exhibited an increase in tensile modulus.



Figure 13. Comparison between weight and tensile modulus of PLA 3D-printed samples before and after oven temperature treatment.

\subsubsection{Flexural Test Results}

The result of a flexural test displays the flexural modulus and weight difference of the 3D-printed samples in Figure 14. There was, on general, a 0.2\% reduction in weight of the specimens after the oven temperature treatment process. The concentric patterns improved by $+13 \%$ in the flexural modulus. However, the rectilinear patterns did not perform as estimated, although they showed an increase in modulus by $+4 \%$. 


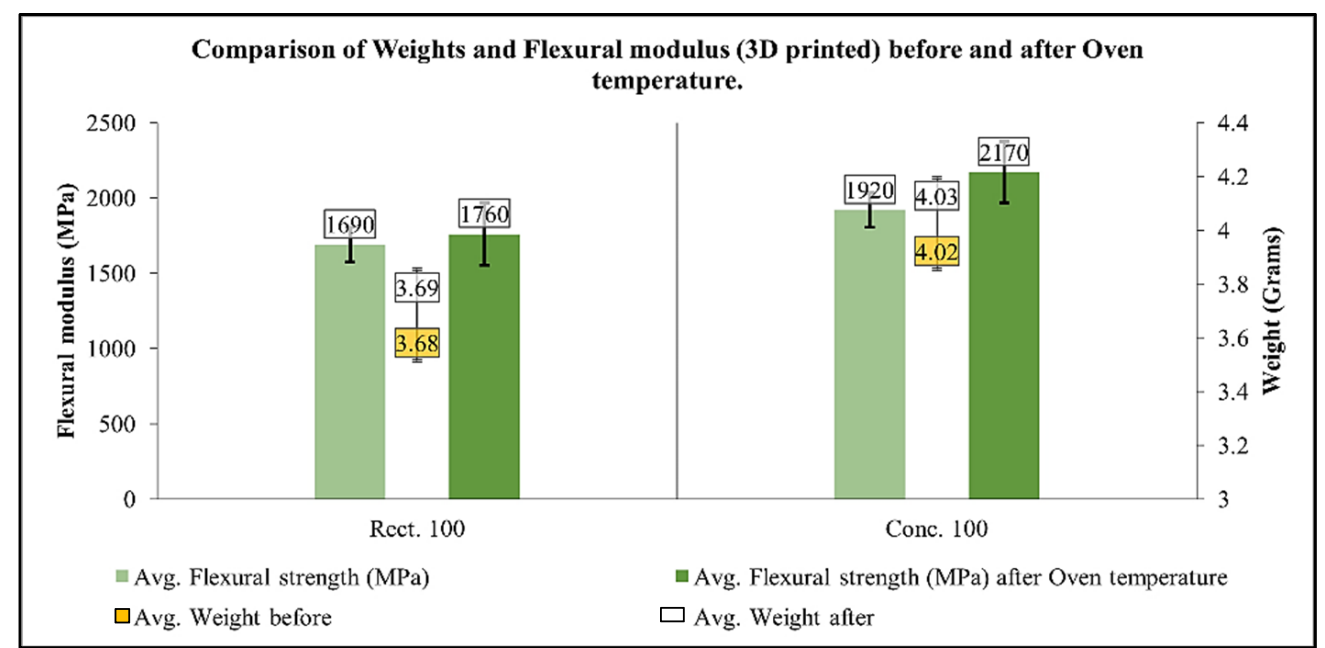

Figure 14. Comparison between weights and flexural modulus of PLA 3D-printed samples before and after oven temperature treatment.

\subsubsection{Charpy Impact Test Results}

The results of the impact test display the impact strength of the 3D-printed samples in Figure 15; there was, on general, a $0.2 \%$ reduction in the weight of the specimens after oven temperature treatment process. Both patterns shown an excellent increment in impact strength.

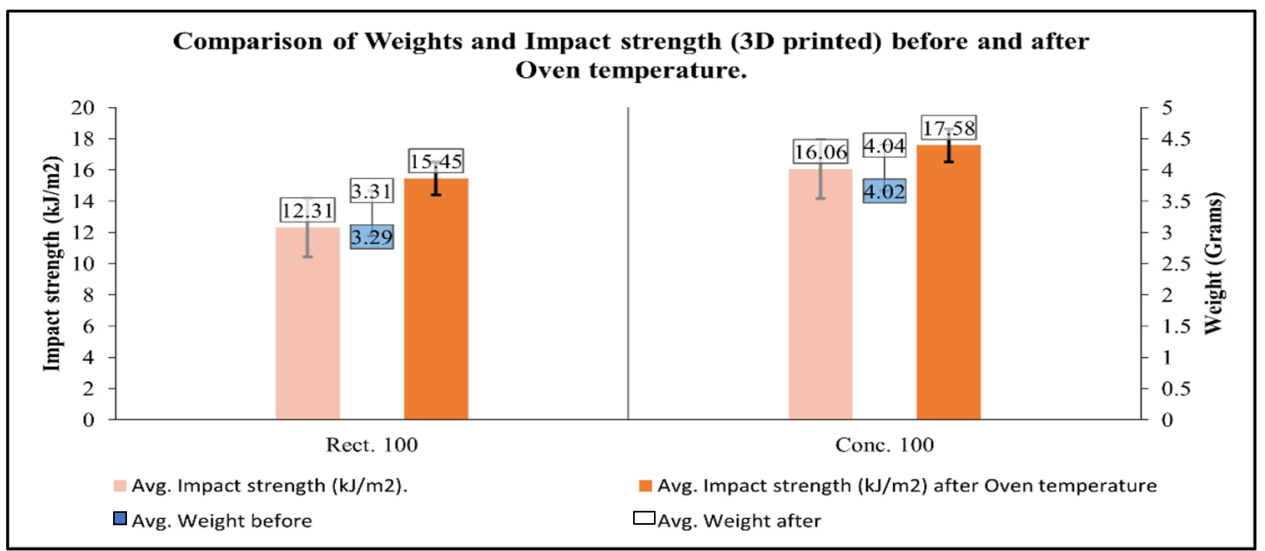

Figure 15. Comparison between weights and impact strength of PLA 3D-printed samples before and after oven temperature treatment.

\subsection{Comparing the 3D-Printed PLA before and after Autoclave Pressurization \\ 4.3.1. Tensile Test Results}

The result of a tensile test displays the tensile modulus and weight difference of the 3D-printed samples in Figure 16, there was on general of $0.25 \%$ increase in weight of the specimens after autoclave pressurization treatment process. The rectilinear infill patterns show an increase of $+40 \%$ in tensile modulus. On the other hand, the concentric specimens showed a decrease in modulus by $-10 \%$. 


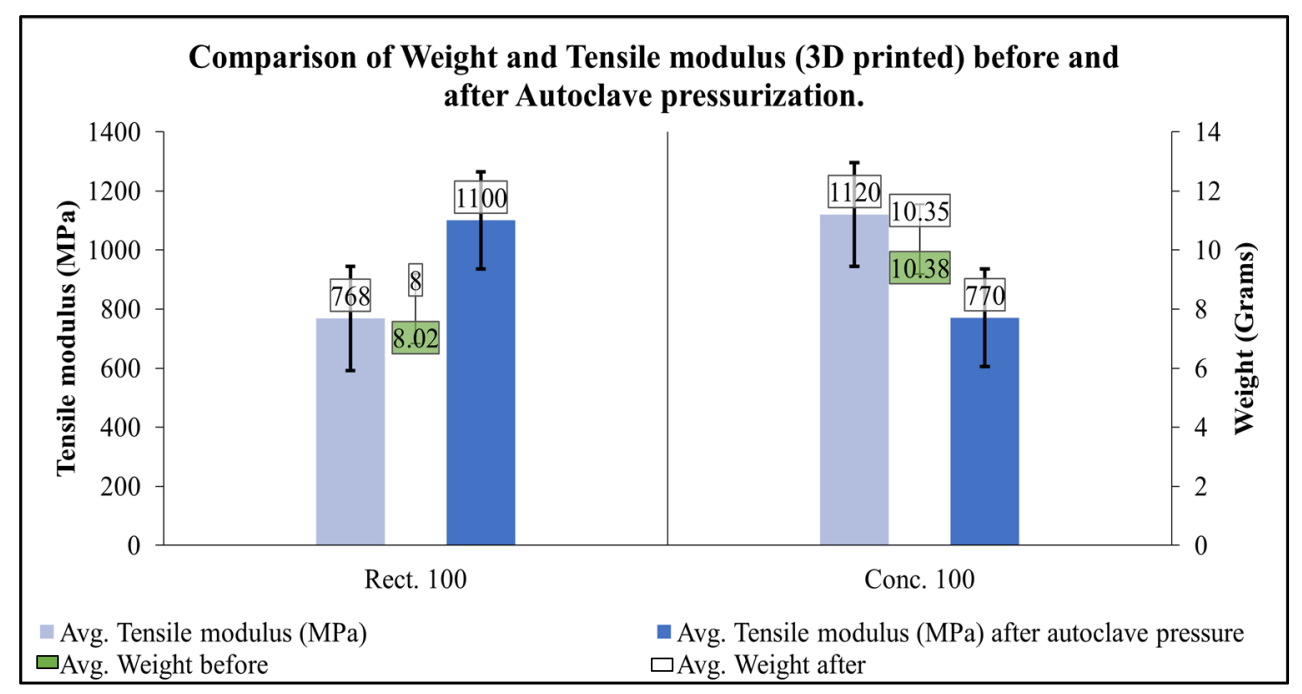

Figure 16. Showing comparison between weights and tensile modulus of PLA 3D-printed samples before and after autoclave pressurization.

\subsubsection{Flexural Test Results}

The result of a flexural test displays the flexural modulus and weight difference of the 3D-printed samples in Figure 17; there was, on general, a $0.2 \%$ increase in weight of the specimens after autoclave pressurization treatment process. The rectilinear pattern yields an improvement, but the concentric pattern did not show any change in flexural modulus.

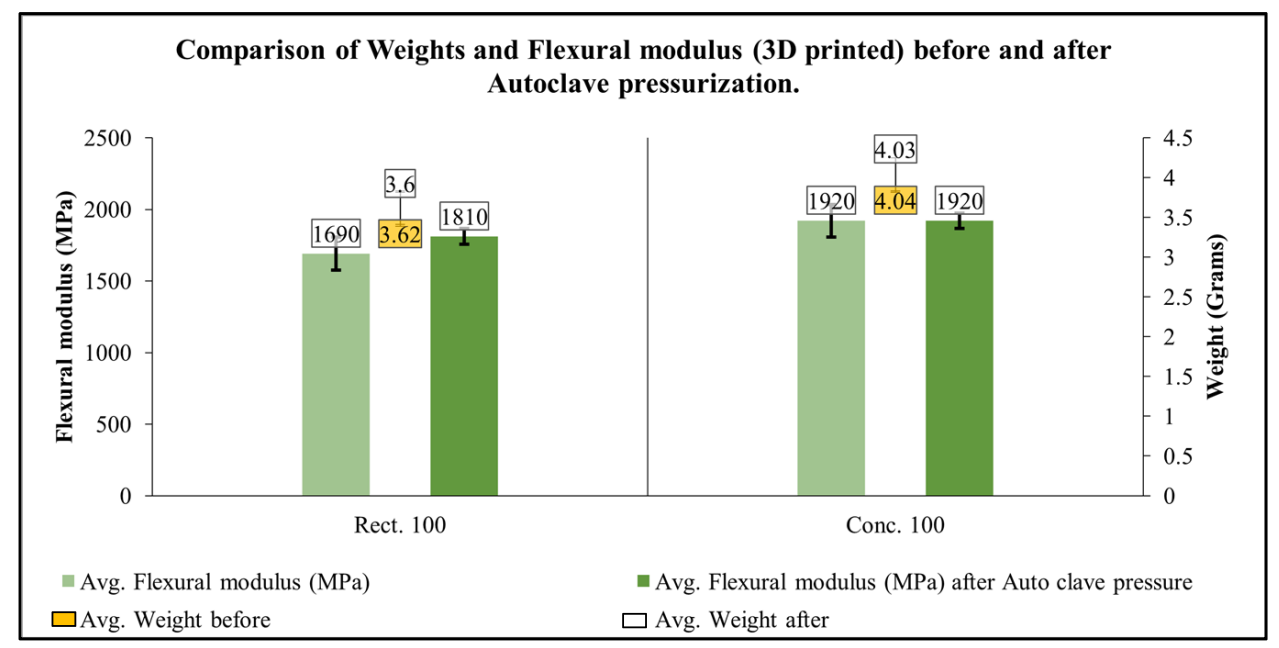

Figure 17. Showing comparison between weights and flexural modulus of PLA 3D-printed samples before and after autoclave pressurization.

\subsubsection{Charpy Impact Test Results}

The results of impact test display both the properties of the impact strength and weight difference of the 3D-printed samples, as shown in Figure 18; there was, in general, a $0.2 \%$ increase in weight of the specimens after autoclave pressurization treatment process. The rectilinear pattern illustrated an increase by $+10 \%$ in impact strength. The concentric pattern did not significantly improve. 


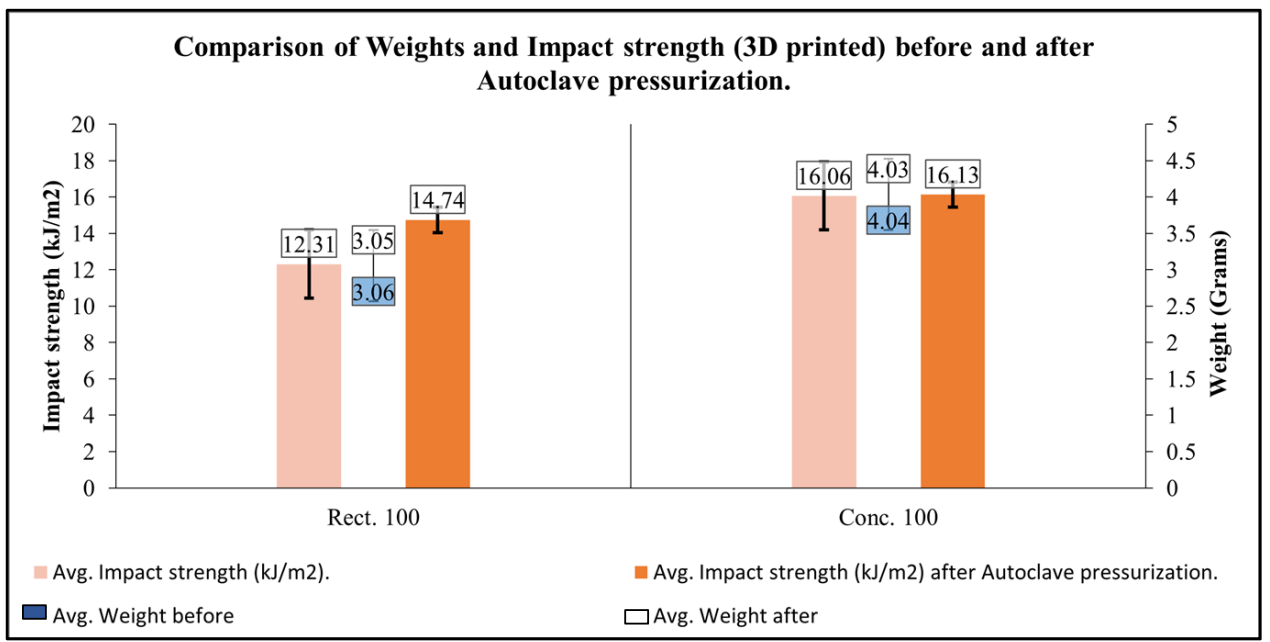

Figure 18. Comparison between weights and impact strength of PLA 3D-printed samples before and after autoclave pressurization.

\section{Conclusions}

Autoclaving pressure and temperature treatment has certainly improved properties in all categories, such as modulus and strength in injection-molded specimens. This is due to the fact that the post-treatment process releases internal stresses in the samples. In the autoclave, samples absorb moisture from hot compressed air, their internal crystalline structure is reorganized, which resulted in a larger grain structure. This aided in modulus and strength growth.

The 3D-printed samples were placed in an autoclave at temperatures less than or equal to the glass transition temperature; at this point, pressurization helped to prevent warping, improved the consolidation of layers, and nullified any voids. Tensile modulus was greatly increased. However, flexural and impact strength have low effect on the strength of the specimens. The weights also changed due to internal molecular rearrangement.

In the oven temperature treatment, the concentric printing pattern of 3D-printed samples appears to be the best. It has excellent tensile and flexural resistance. After oven temperature treatment, the weights of the dog bone specimens decreased by an average of $0.2 \%$, while the weights of rectangular specimens decreased by an average of $0.25 \%$; justification for this is the pattern's deportation of the moisture from the internal layers in the oven temperature treatment process.

In autoclave pressurization of 3D-printed samples, comparing the patterns, the rectilinear infill printing pattern appears to be better. It has an excellent tensile modulus: the rectilinear pattern has an increase of 50\%, whereas the concentric pattern was decreased by an average of $15 \%$. The reasons for the change are unknown. Flexural modulus increased by $7 \%$ for both the rectilinear and concentric patterns, whereas concentric pattern did not show any difference. Impact strength had an increase of $13 \%$ and $4 \%$, respectively, for concentric and rectilinear patterns. This is due to the pattern's absorption of the moisture, which resulted in elevated elastic deformation of the PLA.

Broadly speaking, the oven temperature-treated specimens showed greater modulus and strength gains than the autoclave-pressurized specimens. The weight of the specimen had some positive impact on the properties of the material. However, the concentric infill pattern was almost $90 \%$ nearer to that of the injection-molded specimens' tensile, flexural modulus, and impact strength.

Author Contributions: Y.P.S. and M.M. contributed to the data collection, synthesis and writing of the initial drafts of the manuscript. J.S. helped with research progress and draft revisions. A.S. and H.R.K. helped in analysis of test results. All authors have read and agreed to the published version of the manuscript. 
Funding: This research received no external funding.

Institutional Review Board Statement: Not applicable.

Informed Consent Statement: Not applicable.

Data Availability Statement: Not applicable.

Acknowledgments: Author would like to thank Jens Schuster for providing laboratory equipment and its facilities at University of Applied Sciences Kaiserslautern, Geramny \& IKW.

Conflicts of Interest: The authors declare no conflict of interest.

\section{References}

1. Ning, F.; Cong, W.; Qiu, J.; Wei, J.; Wang, S. Additive manufacturing of carbon fiber reinforced thermoplastic composites using fused deposition modeling. Elseiver Compos. Part B 2015, 80, 369-378. [CrossRef]

2. Schirmeistera, C.G.; Heesa, T.; Lichtb, E.H.; Mülhaupt, R. 3D printing of high density polyethylene by fused filament fabrication. Addit. Manuf. 2019, 28, 152-159. [CrossRef]

3. Shaik, Y.P.; Jens, S.; Ram Chowdary, T. Impact of 3d printing patterns and post consolidation pressure on mechanical properties of FDM 3D printed samples. Am. Res. J. 2021, 2, 1-10.

4. MANUFACTUR3D. How FDM/FFF 3D Printing Technology Works? MANUFACTUR3D. 2018. Available online: https:// manufactur3dmag.com/working-fdm-fff-3d-printing-technology/ (accessed on 15 August 2021).

5. Lederle, F.; Meyer, F.; Brunotte, G.P.; Kaldun, C.; Hübner, E.G. Improved mechanical properties of 3D-printed parts by fused deposition modeling processed under the exclusion of oxygen. Progress. Adiitive Manuf. 2016, 1, 3-7. [CrossRef]

6. Butt, J.; Bhaskar, R. Investigating the Effects of Annealing on the Mechanical Properties of FFF-Printed Thermoplastics. J. Manuf. Mater. Process. 2020, 4, 38. [CrossRef]

7. Wang, Y.; Liu, Z.; Gu, H.; Cui, C.; Hao, J. Improved mechanical properties of 3D-printed SiC/PLA composite parts by microwave heating. J. Mater. Res. 2019, 34, 3412-3419. [CrossRef]

8. Dawoud, M.; Taha, I.; Ebeid, S. Mechanical behaviour of ABS: An experimental study using FDM and injection moulding. $J$. Manuf. Process. 2016, 21, 39-45. [CrossRef]

9. Fabbmatic. MendelMax. Available online: http://www.fabbmatic.com/de_DE (accessed on 30 June 2021).

10. Rep RapWorld, REAL FILAMENT. RepRapWorld B.V. Netherlands. Available online: https://reprapworld.co.uk/listing/ filament/pla/ (accessed on 15 June 2021).

11. ALL3DP. Available online: https:/ / all3dp.com/1/pla-filament-3d-printing/ (accessed on 24 June 2021).

12. Ventola, C.L. Medical Applications for 3D Printing: Current and Projected Uses. Pharm.Ther. 2014, $39,704$.

13. Prakash, K.S.; Nancharaih, T.; Rao, V.S. Additive Manufacturing Techniques in Manufacturing-An Overview. Proceedings 2018, 5 , 3873-3882. [CrossRef]

14. Lopes, M.S.; Jardini, A.L.; Maciel Filho, R.J.P.E. Poly (Lactic Acid) Production for Tissue Engineering Applications. Procedia Eng. 2012, 42, 1402-1413. [CrossRef]

15. Noorani, R. 3D Printing: Technology, Applications and Selection; CRC Press: Boca Raton, FL, USA, 2018.

16. Huang, J.D.X.; Muley, P.; Wu, T.; Barekati-Goudarzi, M.; Tang, Z.; Li, M.; Lee, S.; Boldor Dand Wu, Q. Carbonized cellulose nanofibers as dielectric heat sources for microwave annealing 3D printed PLA composite. Compos. Part B Eng. 2020, 184, 107640.

17. Ayrilmis, N.; Kariz, M.; Kwon, J.H.; Kitek Kuzman, M. Effect of Printing Layer Thickness on Water Absorption and Mechanical Properties of 3D-Printed Wood/PLA Composite Materials. Int. J. Adv. Manuf. Technol. 2019, 102, 2195-2200. [CrossRef]

18. Custompartnet.net. Injection Molding. Available online: http://www.custompartnet.com/wu/InjectionMolding\#materials (accessed on 1 June 2021).

19. Introduction to Injection Molding, Predictabledesigns.com. Available online: https://predictabledesigns.com/introduction-toinjection-molding/ (accessed on 7 September 2021).

20. Arburg. Available online: https:/ /www.arburg.com/en/ (accessed on 10 April 2021).

21. Haage Anagaram Technologien GmbH. Available online: http://www.haage.com/EN/products/high-pressure-autoclaves/ (accessed on 20 August 2021).

22. Firstsource. A Comprehensive Guide to Know Autoclave and Its Usage. Available online: https://www.firstsourcels.com/blog/ a-comprehensive-guide-to-know-autoclave-and-its-usage/ (accessed on 16 August 2021).

23. Sterilization of Liquids. Solids. Waste in Disposal Bags and Hazardous Biological Substances. Systec the Autoclave Company. Available online: https://www.systec-lab.com/news-events/sterilization-of-liquids-solids-waste-in-disposal-bags-andhazardous-biological-substances / (accessed on 19 October 2021).

24. Simpson, R.B. Rubber Basics. Available online: https://docplayer.net/185279198-Rubber-basics-edited-by-r-b-simpson.html (accessed on 19 October 2021). 